\title{
A further remark to paper 'Convergence theorems for the common solution for a finite family of $\phi$-strongly accretive operator equations'
}

\section{Zhiqun Xue ${ }^{1 *}$ and Haiyun Zhou ${ }^{2}$}

${ }^{*}$ Correspondence:

xuezhiqun@126.com

${ }^{1}$ Department of Mathematics and

Physics, Shijiazhuang Tiedao

University, Shijiazhuang, 050043,

China

Full list of author information is

available at the end of the article

\begin{abstract}
In this note, we point out several gaps in Gurudwan and Sharma (Appl. Math. Comput. 217(15):6748-6754, 2011) and Yang (Appl. Math. Comput.

218(21):10367-10369, 2012) and give the main results under weaker conditions.

MSC: 47H10;47H09; 46B20
\end{abstract}

Keywords: uniformly continuous; $\Phi$-strongly accretive; multi-step iteration with errors; Banach space

\section{Introduction}

Recently, Gurudwan, Sharma [1] and Yang [2] studied the strong convergence of the sequence, respectively, which was defined by

$$
\begin{aligned}
& x_{0} \in E, \\
& x_{n}^{1}=a_{n}^{1} x_{n}+b_{n}^{1} S_{1} x_{n}+c_{n}^{1} u_{n}^{1}, \\
& x_{n}^{2}=a_{n}^{2} x_{n}+b_{n}^{2} S_{2} x_{n}^{1}+c_{n}^{2} u_{n}^{2}, \\
& \vdots \\
& x_{n+1}=x_{n}^{N}=a_{n}^{N} x_{n}+b_{n}^{N} S_{N} x_{n}^{N-1}+c_{n}^{N} u_{n}^{N}, \quad n \geq 0,
\end{aligned}
$$

for approximation of a common solution of a finite family of uniformly continuous $\Phi$-strongly accretive operator equations. Their results are as follows.

Theorem GS [1, Theorem 3.1] Let E be an arbitrary real Banach space and let $\left\{A_{i}\right\}_{i=1}^{N}$ : $E \rightarrow E$ be uniformly continuous $\phi$-strongly accretive operators and each range of either $A_{i}$ or $\left(I-A_{i}\right)$ be bounded. Let, for $i=1, \ldots, N,\left\{u_{n}^{i}\right\}_{n=1}^{\infty}$ be sequences in $E$ and $\left\{a_{n}^{i}\right\}_{n=1}^{\infty},\left\{b_{n}^{i}\right\}_{n=1}^{\infty}$, $\left\{c_{n}^{i}\right\}_{n=1}^{\infty}$ be real sequences in $[0,1]$ satisfying
(i) $a_{n}^{i}+b_{n}^{i}+c_{n}^{i}=1$,
(ii) $\sum_{n=0}^{\infty} b_{n}^{N}=\infty$,
(iii) $\sum_{n=0}^{\infty} c_{n}<\infty$,
(iv) $\lim _{n \rightarrow \infty} b_{n}^{i}=\lim _{n \rightarrow \infty} c_{n}^{i}=\lim _{n \rightarrow \infty} \frac{c_{n}^{i}}{b_{n}^{i}+c_{n}^{i}}=0, \forall i=1, \ldots, N, n \geq 1$.

( 2013 Xue and Zhou; licensee Springer. This is an Open Access article distributed under the terms of the Creative Commons Attribution License (http://creativecommons.org/licenses/by/2.0), which permits unrestricted use, distribution, and reproduction in any medium, provided the original work is properly cited. 
For any given $f \in E$, define $\left\{S_{i}\right\}_{i=1}^{N}: E \rightarrow E$ by $S_{i} x=x-A_{i} x+f, \forall i=1, \ldots, N, \forall x \in E$. Then the multi-step iterative sequence with errors $\left\{x_{n}\right\}_{n=1}^{\infty}$ defined by the above converges strongly to the unique solution of the operator equations $\left\{A_{i} x\right\}_{i=1}^{N}=f$.

On the basis of the above result, Yang [2] proved the following convergence theorem.

Thoerem Yang [2, Theorem 2] Let $E$ be an arbitrary real Banach space and let $\left\{A_{i}\right\}_{i=1}^{N}$ : $E \rightarrow E$ be uniformly continuous $\phi$-strongly accretive operators and each range of either $A_{i}$ or $\left(I-A_{i}\right)$ is bounded. Let for $i=1, \ldots, N,\left\{u_{n}^{i}\right\}_{n=1}^{\infty}$ be bounded sequences in $E$ and $\left\{a_{n}^{i}\right\}_{n=1}^{\infty}$, $\left\{b_{n}^{i}\right\}_{n=1}^{\infty},\left\{c_{n}^{i}\right\}_{n=1}^{\infty}$ be real sequences in $[0,1]$ satisfying

(i) $a_{n}^{i}+b_{n}^{i}+c_{n}^{i}=1$

(ii) $\sum_{n=0}^{\infty} b_{n}^{N}=\infty$,

(iii) $\lim _{n \rightarrow \infty} b_{n}^{i}=\lim _{n \rightarrow \infty} c_{n}^{i}=\lim _{n \rightarrow \infty} \frac{c_{n}^{i}}{b_{n}^{i}+c_{n}^{i}}=0, \forall i=1, \ldots, N, n \geq 1$.

For any given $f \in E$, define $\left\{S_{i}\right\}_{i=1}^{N}: E \rightarrow E$ by $S_{i} x=\left(I-A_{i}\right) x+f, \forall i=1, \ldots, N, \forall x \in E$. Then the multi-step iterative sequence with errors $\left\{x_{n}\right\}_{n=1}^{\infty}$ defined by the above converges strongly to the unique solution of the operator equations $\left\{A_{i} x\right\}_{i=1}^{N}=f$.

However, after careful reading of their works, we discovered that there exist some problems in references [1] and [2] as follows.

Problem 1 In the proof course of Theorem 3.1 of Gurudwan and Sharma [1], which happens in line 11 of page 6751 . Here, it is defective that they obtained $\|x-y\| \leq \phi_{i}^{-1}\left(\| A_{i} x-\right.$ $\left.A_{i} y \|\right)$, that is, $\left\langle A_{i} x-A_{i} y, j(x-y)\right\rangle \geq \phi(\|x-y\|)\|x-y\| \Rightarrow \phi(\|x-y\|) \leq\left\|A_{i} x-A_{i} y\right\|$, but we cannot deduce $\|x-y\| \leq \phi_{i}^{-1}\left(\left\|A_{i} x-A_{i} y\right\|\right)$. The reason is that it is possible $\left\|A_{i} x-A_{i} y\right\|$ does not belong to $R(\phi)$ (range of $\phi$ ). A counterexample is as follows. Let us define $\phi:[0,+\infty) \rightarrow[0,+\infty)$ by $\phi(\alpha)=\frac{2^{\alpha}-1}{2^{\alpha}+1}$; then it can be easily seen that $\phi$ is increasing with $\phi(0)=0$, but $\lim _{\alpha \rightarrow+\infty} \phi(\alpha)=1$ and $\phi^{-1}(2)$ makes no sense (see [3]).

Problem 2 In the paper of Yang [2], he referred to the mistakes of ${ }^{\prime}\left\|x_{n_{m}+j}^{i}-q\right\|<\epsilon$ for $j \geq 1$ to deduce $\left\|x_{n}-q\right\| \rightarrow 0(n \rightarrow \infty)$ ' in [1] and cited an example, i.e.,

$$
\left\{v_{n}\right\}=\{1,0,2,0,0,3,0,0,0,4,0,0,0,0,5,0,0,0,0,0,6,0,0, \ldots\} .
$$

Now, we want to clarify the fact. Let $\left\{\gamma_{n}\right\}$ be a real sequence, $\left\{\gamma_{n_{m}}\right\}$ be some infinite subsequence of $\left\{\gamma_{n}\right\}$ and $\left\{n_{m}\right\}$ be neither odd nor even sequence, then the conclusions are as follows:

(C-i) $\lim _{n \rightarrow \infty} \gamma_{n}=0 \Leftrightarrow \forall \epsilon>0, \exists$ nonnegative integer $n_{0}$ such that $\left|\gamma_{n_{m}+j}\right|<\epsilon$ for $n_{m} \geq n_{0}, j \geq 1$.

(C-ii) $\lim _{n \rightarrow \infty} \gamma_{n}=0 \Rightarrow \lim _{m \rightarrow \infty} \gamma_{n_{m}}=0$ and $\lim _{m \rightarrow \infty} \gamma_{n_{m}+j}=0$ for $\forall j \geq 1$.

Indeed, the above example $\left.{ }^{* * *}\right)$ does not satisfy the conclusion $(\mathrm{C}-\mathrm{i})$, it just illustrates the result ( $\mathrm{C}$-ii). Therefore, the note given by Yang [2] confused the conclusions $(\mathrm{C}-\mathrm{i})$ and (C-ii).

The aim of this paper is to generalize the results of papers [1] and [2]. For this, we need the following knowledge. 


\section{Preliminary}

Let $E$ be a real Banach space and $E^{*}$ be its dual space. The normalized duality mapping $J: E \rightarrow 2^{E^{*}}$ is defined by

$$
J(x)=\left\{f \in E^{*}:\langle x, f\rangle=\|x\|^{2}=\|f\|^{2}\right\}, \quad \forall x \in E,
$$

where $\langle\cdot, \cdot\rangle$ denotes the generalized duality pairing. The single-valued normalized duality mapping is denoted by $j$.

An operator $T: E \rightarrow E$ is said to be strongly accretive if there exists a constant $k>0$, and for $\forall x, y \in E, \exists j(x-y) \in J(x-y)$ such that

$$
\langle T x-T y, j(x-y)| \geq k\|x-y\|^{2},
$$

without loss of generality, we assume that $k \in(0,1)$. The operator $T$ is called $\phi$-strongly accretive if for any $x, y \in E$, there exist $j(x-y) \in J(x-y)$ and a strictly increasing continuous function $\phi:[0,+\infty) \rightarrow[0,+\infty)$ with $\phi(0)=0$ such that

$$
\langle T x-T y, j(x-y)\rangle \geq \phi(\|x-y\|)\|x-y\| .
$$

It is obvious that a strongly accretive operator must be the $\phi$-strongly accretive in the special case in which $\phi(t)=k t$, but the converse is not true in general. That is, the class of strongly accretive operators is a proper subclass of the class of $\phi$-strongly accretive operators.

In order to obtain the main conclusion of this paper, we need the following lemmas.

Lemma 2.1 [1] Suppose that $E$ is an arbitrary Banach space and $A: E \rightarrow E$ is a continuous $\phi$-strongly accretive operator. Then the equation $A x=f$ has a unique solution for any $f \in E$.

Lemma 2.2 [4] Let E be a real Banach space and let $J: E \rightarrow 2^{E^{*}}$ be a normalized duality mapping. Then

$$
\|x+y\|^{2} \leq\|x\|^{2}+2\langle y, j(x+y)\rangle
$$

for all $x, y \in E$ and $j(x+y) \in J(x+y)$.

Lemma 2.3 [5] Let $\left\{\delta_{n}\right\}_{n=0}^{\infty},\left\{\lambda_{n}\right\}_{n=0}^{\infty}$ and $\left\{\gamma_{n}\right\}_{n=0}^{\infty}$ be three nonnegative real sequences and $\phi$ : $[0,+\infty) \rightarrow[0,+\infty)$ be a strictly increasing and continuous function with $\phi(0)=0$ satisfying the following inequality:

$$
\delta_{n+1}^{2} \leq \delta_{n}^{2}-\lambda_{n} \phi\left(\delta_{n+1}\right)+\gamma_{n}, \quad n \geq 0,
$$

where $\lambda_{n} \in[0,1]$ with $\sum_{n=0}^{\infty} \lambda_{n}=\infty, \gamma_{n}=o\left(\lambda_{n}\right)$. Then $\delta_{n} \rightarrow 0$ as $n \rightarrow \infty$.

\section{Main results}

Theorem 3.1 Let $E$ be an arbitrary real Banach space and $\left\{A_{i}\right\}_{i=1}^{N}: E \rightarrow E$ be $N$ uniformly continuous $\phi$-strongly accretive operators. For $i=1,2, \ldots, N$, let $\left\{u_{n}^{i}\right\}_{n=1}^{\infty}$ be bounded sequences in $E$ and $\left\{a_{n}^{i}\right\}_{n=1}^{\infty},\left\{b_{n}^{i}\right\}_{n=1}^{\infty},\left\{c_{n}^{i}\right\}_{n=1}^{\infty}$ be real sequences in $[0,1]$ satisfying 
(i) $a_{n}^{i}+b_{n}^{i}+c_{n}^{i}=1, i=1,2, \ldots, N$;

(ii) $\sum_{n=1}^{\infty} b_{n}^{N}=+\infty$;

(iii) $\lim _{n \rightarrow \infty} b_{n}^{i}=\lim _{n \rightarrow \infty} c_{n}^{i}=0, i=1,2, \ldots, N$;

(iv) $c_{n}^{N}=o\left(b_{n}^{N}\right)$.

For any given $f \in E$, define $\left\{S_{i}\right\}_{i=1}^{N}: E \rightarrow E$ with $\bigcap_{i=1}^{N} F\left(S_{i}\right) \neq \emptyset$ by $S_{i} x=x-A_{i} x+f, \forall i=$ $1,2, \ldots, N, \forall x \in E$, where $F\left(S_{i}\right)=\left\{x \in E: S_{i} x=x\right\}$. Then, for some $x_{0} \in E$, the multi-step iterative sequence with errors $\left\{x_{n}\right\}_{n=1}^{\infty}$ defined by

$$
\begin{aligned}
& x_{0} \in E, \\
& x_{n}^{1}=a_{n}^{1} x_{n}+b_{n}^{1} S_{1} x_{n}+c_{n}^{1} u_{n}^{1}, \\
& x_{n}^{2}=a_{n}^{2} x_{n}+b_{n}^{2} S_{2} x_{n}^{1}+c_{n}^{2} u_{n}^{2}, \\
& \vdots \\
& x_{n}^{N-1}=a_{n}^{N-1} x_{n}+b_{n}^{N-1} S_{N-1} x_{n}^{N-2}+c_{n}^{N-1} u_{n}^{N-1}, \\
& x_{n+1}=x_{n}^{N}=a_{n}^{N} x_{n}+b_{n}^{N} S_{N} x_{n}^{N-1}+c_{n}^{N} u_{n}^{N}, \quad n \geq 0,
\end{aligned}
$$

converges strongly to the unique solution of the operator equations $\left\{A_{i} x\right\}_{i=1}^{N}=f$.

Proof Since $\left\{A_{i}\right\}_{i=1}^{N}: E \rightarrow E$ is $\phi$-strongly accretive operator, we obtain that each equation $A_{i} x=f$ has the unique solution by Lemma 2.1, denote $q_{i}$, i.e., $q_{i}$ is the unique fixed point of $S_{i}$ by $S_{i} x=x-A_{i} x+f$. Since $\bigcap_{i=1}^{N} F\left(S_{i}\right) \neq \emptyset$, then $\bigcap_{i=1}^{N} F\left(S_{i}\right)$ is a single set, let $q$. Meanwhile, there exists a strictly increasing continuous function $\phi:[0,+\infty) \rightarrow[0,+\infty)$ with $\phi(0)=0$ such that

$$
\left\langle A_{i} x-A_{i} q, j(x-q)\right\rangle \geq \phi(\|x-q\|)
$$

for $x \in E, q \in F(T)$, that is,

$$
\left\langle S_{i} x-x, j(x-q)\right\rangle \leq-\phi(\|x-q\|)
$$

Choose some $x_{0} \in E$ and $x_{0} \neq S_{i} x_{0}$ such that $r_{0} \in R(\Phi)$, where

$$
r_{0}=\max \left\{\left\|x_{0}-S_{1} x_{0}\right\| \cdot\left\|x_{0}-q\right\|,\left\|x_{0}-S_{2} x_{0}\right\| \cdot\left\|x_{0}-q\right\|, \ldots,\left\|x_{0}-S_{N} x_{0}\right\| \cdot\left\|x_{0}-q\right\|\right\},
$$

$R(\Phi)$ is the range of $\Phi$. Indeed, if $\Phi(r) \rightarrow+\infty$ as $r \rightarrow+\infty$, then $r_{0} \in R(\Phi)$; if $\sup \{\Phi(r): r \in$ $[0,+\infty)\}=r_{1}<+\infty$ with $r_{1}<r_{0}$, then for $q \in E$, there exists a sequence $\left\{w_{n}\right\}$ in $E$ such that $w_{n} \rightarrow q$ as $n \rightarrow \infty$ with $w_{n} \neq q$. Since $A_{i}$ is uniformly continuous, so is $S_{i}$. Furthermore, we obtain that $S_{i} w_{n} \rightarrow S_{i} q$ as $n \rightarrow \infty$, then $\left\{w_{n}-S_{i} w_{n}\right\}$ is the bounded sequence for $i=$ $1,2, \ldots, N$. Hence, there exists the common natural number $n_{0}$ such that $\left\|w_{n}-S_{i} w_{n}\right\| \cdot \| w_{n}-$ $q \|<\frac{r_{1}}{2}$ for $n \geq n_{0}$ and $i=1,2, \ldots, N$, then we redefine $x_{0}=w_{n_{0}}$ and $\left\|x_{0}-S_{i} x_{0}\right\| \cdot\left\|x_{0}-q\right\|<\frac{r_{1}}{2}$. Thus, $\max _{1 \leq i \leq N}\left\{\left\|x_{0}-S_{i} x_{0}\right\| \cdot\left\|x_{0}-q\right\|\right\} \in R(\phi)$. It is to ensure that $\Phi^{-1}\left(r_{0}\right)$ is defined well.

Step I. We show that $\left\{x_{n}\right\}$ is a bounded sequence.

Set $R=\Phi^{-1}\left(r_{0}\right)$, then from the above formula (@), we obtain that $\left\|x_{0}-q\right\| \leq R$. Denote

$$
B_{1}=\{x \in E:\|x-q\| \leq R\}, \quad B_{2}=\{x \in E:\|x-q\| \leq 2 R\} .
$$


Since $S_{i}$ is uniformly continuous, then $S_{i}$ is bounded. We let

$$
M=\max _{1 \leq i \leq N}\left\{\sup _{x \in B_{2}}\left\{\left\|S_{i} x-q\right\|+1\right\}\right\}+\max _{1 \leq i \leq N}\left\{\sup _{n}\left\{\left\|u_{n}^{i}-q\right\|\right\}\right\}
$$

Next, we want to prove that $x_{n} \in B_{1}$. If $n=0$, then $x_{0} \in B_{1}$. Now, assume that it holds for some $n$, i.e., $x_{n} \in B_{1}$. We prove that $x_{n+1} \in B_{1}$. Suppose it is not the case, then $\left\|x_{n+1}-q\right\|>$ $R>\frac{R}{2}$. Since $S_{i}$ is uniformly continuous for $i=1,2, \ldots, N$, then for $\epsilon_{0}=\frac{\Phi\left(\frac{R}{2}\right)}{8 R}$, there exists common $\delta>0$ such that $\left\|S_{i} x-S_{i} y\right\|<\epsilon_{0}$ when $\|x-y\|<\delta$. Denote

$$
\tau_{0}=\min \left\{1, \frac{R}{M}, \frac{\Phi\left(\frac{R}{2}\right)}{8 R(M+2 R)}, \frac{\delta}{2 M+5 R}\right\} .
$$

Since $b_{n}^{i}, c_{n}^{i} \rightarrow 0$ as $n \rightarrow \infty$ for $i=1,2, \ldots, p$. Without loss of generality, we let $0 \leq b_{n}^{i}, c_{n}^{i} \leq$ $\tau_{0}$ for any $n \geq 0$ and $i=1,2, \ldots, N$. Since $c_{n}^{N}=o\left(b_{n}^{N}\right)$, let $c_{n}^{N}<b_{n}^{N} \tau_{0}$. Now, estimate $\left\|x_{n}^{i}-q\right\|$ for $i=1,2, \ldots, N$. From the multi-step iteration, we have

$$
\begin{aligned}
& \left\|x_{n}^{1}-q\right\| \\
& \quad \leq\left(1-b_{n}^{1}-c_{n}^{1}\right)\left\|x_{n}-q\right\|+b_{n}^{1}\left\|S_{1} x_{n}-q\right\|+c_{n}^{1}\left\|u_{n}^{1}-q\right\| \\
& \quad \leq R+\tau_{0} M \\
& \quad \leq 2 R
\end{aligned}
$$

then $x_{n}^{1} \in B_{2}$. Similarly, we have

$$
\begin{aligned}
& \left\|x_{n}^{2}-q\right\| \\
& \quad \leq\left(1-b_{n}^{2}-c_{n}^{2}\right)\left\|x_{n}-q\right\|+b_{n}^{2}\left\|S_{2} x_{n}^{1}-q\right\|+c_{n}^{2}\left\|u_{n}^{2}-q\right\| \\
& \quad \leq R+\tau_{0} M \\
& \quad \leq 2 R
\end{aligned}
$$

then $x_{n}^{2} \in B_{2} . \cdots \cdots$, we have

$$
\begin{aligned}
& \left\|x_{n}^{N-1}-q\right\| \\
& \quad \leq\left(1-b_{n}^{N-1}-c_{n}^{N-1}\right)\left\|x_{n}-q\right\|+b_{n}^{N-1}\left\|S_{N-1} x_{n}^{N-2}-q\right\|+c_{n}^{N-1}\left\|u_{n}^{N-1}-q\right\| \\
& \quad \leq R+\tau_{0} M \\
& \quad \leq 2 R
\end{aligned}
$$

then $x_{n}^{N-1} \in B_{2}$. Therefore, we get

$$
\begin{aligned}
& \left\|x_{n+1}-q\right\| \\
& \quad \leq\left(1-b_{n}^{N}-c_{n}^{N}\right)\left\|x_{n}-q\right\|+b_{n}^{N}\left\|S_{N} x_{n}^{N-1}-q\right\|+c_{n}^{N}\left\|u_{n}^{N}-q\right\| \\
& \quad \leq R+\tau_{0} M \\
& \quad \leq 2 R .
\end{aligned}
$$


And we also have

$$
\begin{aligned}
& \left\|x_{n+1}-x_{n}\right\| \\
& \quad \leq b_{n}^{N}\left\|S_{N} x_{n}^{N-1}-x_{n}\right\|+c_{n}^{N}\left\|u_{n}^{N}-x_{n}\right\| \\
& \quad \leq b_{n}^{N}\left(\left\|S_{N} x_{n}^{N-1}-q\right\|+\left\|x_{n}-q\right\|\right)+c_{n}^{N}\left(\left\|u_{n}^{N}-q\right\|+\left\|x_{n}-q\right\|\right) \\
& \leq \tau_{0}\left[\left(\left\|S_{N} x^{N-1}-q\right\|+\left\|u_{n}^{N}-q\right\|\right)+2\left\|x_{n}-q\right\|\right] \\
& \leq \tau_{0}(M+2 R) \\
& \quad \leq \frac{\Phi\left(\frac{R}{2}\right)}{8 R},
\end{aligned}
$$

and

$$
\begin{aligned}
&\left\|x_{n+1}-x_{n}^{N-1}\right\| \\
& \leq b_{n}^{N}\left\|S_{N} x_{n}^{N-1}-x_{n}\right\|+c_{n}^{N}\left\|u_{n}^{N}-x_{n}\right\|+b_{n}^{N-1}\left\|S_{N-1} x_{n}^{N-2}-x_{n}\right\|+c_{n}^{N-1}\left\|u_{n}^{N-1}-x_{n}\right\| \\
& \leq b_{n}^{N}\left(\left\|S_{N} x_{n}^{N-1}-q\right\|+\left\|x_{n}-q\right\|\right)+c_{n}^{N}\left(\left\|u_{n}^{N}-q\right\|+\left\|x_{n}-q\right\|\right) \\
& \quad+b_{n}^{N-1}\left(\left\|S_{N-1} x_{n}^{N-2}-q\right\|+\left\|x_{n}-q\right\|\right)+c_{n}^{N-1}\left(\left\|u_{n}^{N-1}-q\right\|+\left\|x_{n}-q\right\|\right) \\
& \leq \tau_{0}\left[\left(\left\|S_{N} x_{n}^{N-1}-q\right\|+\left\|u_{n}^{N}-q\right\|+2\left\|x_{n}-q\right\|\right)\right. \\
&\left.\quad+\left(\left\|S_{N-1} x_{n}^{N-2}-q\right\|+\left\|u_{n}^{N-1}-q\right\|+2\left\|x_{n}-q\right\|\right)\right] \\
& \leq \tau_{0}(2 M+4 R) \\
&< \delta .
\end{aligned}
$$

By the uniform continuity of $S_{N}$, we have

$$
\left\|S_{N} x_{n+1}-S_{N} x_{n}^{N-1}\right\|<\frac{\Phi\left(\frac{R}{2}\right)}{8 R} .
$$

Using Lemma 2.2 and the above formulas, we have

$$
\begin{aligned}
&\left\|x_{n+1}-q\right\|^{2} \\
&=\left\|\left(x_{n}-q\right)+b_{n}^{N}\left(S_{N} x_{n}^{N-1}-x_{n}\right)+c_{n}^{N}\left(u_{n}^{N}-x_{n}\right)\right\|^{2} \\
& \leq\left\|x_{n}-q\right\|^{2}+2 b_{n}^{N}\left\langle S_{N} x_{n}^{N-1}-x_{n}, j\left(x_{n+1}-q\right)\right\rangle+2 c_{n}^{N}\left\langle u_{n}^{N}-x_{n}, j\left(x_{n+1}-q\right)\right\rangle \\
& \leq\left\|x_{n}-q\right\|^{2}+2 b_{n}^{N}\left\langle S_{N} x_{n+1}-x_{n+1}+x_{n+1}-x_{n}-S_{N} x_{n+1}+S_{N} x_{n}^{N-1}, j\left(x_{n+1}-q\right)\right\rangle \\
& \quad+2 c_{n}^{N}\left\|u_{n}^{N}-x_{n}\right\| \cdot\left\|x_{n+1}-q\right\| \\
& \leq\left\|x_{n}-q\right\|^{2}-2 b_{n}^{N} \Phi\left(\left\|x_{n+1}-q\right\|\right)+2 b_{n}^{N}\left\|x_{n+1}-x_{n}\right\| \cdot\left\|x_{n+1}-q\right\| \\
&+2 b_{n}^{N}\left\|S_{N} x_{n+1}-S_{N} x_{n}^{N-1}\right\| \cdot\left\|x_{n+1}-q\right\|+2 c_{n}^{N}\left(\left\|u_{n}^{N}-q\right\|+\left\|x_{n}-q\right\|\right)\left\|x_{n+1}-q\right\| \\
& \leq\left\|x_{n}-q\right\|^{2}-2 b_{n}^{N} \Phi\left(\frac{R}{2}\right)+2 b_{n}^{N} \frac{\Phi\left(\frac{R}{2}\right)}{8 R} \cdot 2 R+2 b_{n}^{N} \frac{\Phi\left(\frac{R}{2}\right)}{8 R} \cdot 2 R+2 b_{n}^{N} \tau_{0}(R+M) 2 R \\
& \leq\left\|x_{n}-q\right\|^{2}-b_{n}^{N} \Phi\left(\frac{R}{2}\right)+2 b_{n}^{N} \frac{\Phi\left(\frac{R}{2}\right)}{8 R(M+2 R)}(R+M) 2 R
\end{aligned}
$$




$$
\begin{aligned}
& \leq\left\|x_{n}-q\right\|^{2}-\frac{b_{n}^{N}}{2} \Phi\left(\frac{R}{2}\right) \\
& \leq R^{2}
\end{aligned}
$$

which is a contradiction. So, $x_{n+1} \in B_{1}$, i.e., $\left\{x_{n}\right\}$ is a bounded sequence, from which it follows that $\left\{x_{n}^{1}\right\},\left\{x_{n}^{2}\right\}, \ldots,\left\{x_{n}^{N-1}\right\}$ are all bounded sequences as well.

Step II. We want to prove $\left\|x_{n}-q\right\| \rightarrow 0$ as $n \rightarrow \infty$.

Since $b_{n}^{i}, c_{n}^{i} \rightarrow 0$ as $n \rightarrow \infty$ for $i=1,2, \ldots, N$ and $\left\{x_{n}\right\},\left\{x_{n}^{N-1}\right\}$ are bounded. From (3.5) and (3.6), we obtain

$$
\lim _{n \rightarrow \infty}\left\|x_{n+1}-x_{n}\right\|=0, \quad \lim _{n \rightarrow \infty}\left\|x_{n+1}-x_{n}^{N-1}\right\|=0, \quad \lim _{n \rightarrow \infty}\left\|S_{N} x_{n+1}-S_{N} x_{n}^{N-1}\right\|=0 .
$$

By (3.7), we have

$$
\begin{aligned}
\left\|x_{n+1}-q\right\|^{2} \\
=\left\|\left(x_{n}-q\right)+b_{n}^{N}\left(S_{N} x_{n}^{N-1}-x_{n}\right)+c_{n}^{N}\left(u_{n}^{N}-x_{n}\right)\right\|^{2} \\
\leq\left\|x_{n}-q\right\|^{2}+2 b_{n}^{N}\left\langle S_{N} x_{n}^{N-1}-x_{n}, j\left(x_{n+1}-q\right)\right\rangle+2 c_{n}^{N}\left\langle u_{n}^{N}-x_{n}, j\left(x_{n+1}-q\right)\right\rangle \\
\leq\left\|x_{n}-q\right\|^{2}+2 b_{n}^{N}\left\langle S_{N} x_{n+1}-x_{n+1}+x_{n+1}-x_{n}-S_{N} x_{n+1}+S_{N} x_{n}^{N-1}, j\left(x_{n+1}-q\right)\right\rangle \\
\quad+2 c_{n}^{N}\left\|u_{n}^{N}-x_{n}\right\| \cdot\left\|x_{n+1}-q\right\| \\
\leq\left\|x_{n}-q\right\|^{2}-2 b_{n}^{N} \Phi\left(\left\|x_{n+1}-q\right\|\right)+2 b_{n}^{N}\left\|x_{n+1}-x_{n}\right\| \cdot\left\|x_{n+1}-q\right\| \\
\quad+2 b_{n}^{N}\left\|S_{N} x_{n+1}-S_{N} x_{n}^{N-1}\right\| \cdot\left\|x_{n+1}-q\right\|+2 c_{n}^{N}\left\|u_{n}^{N}-x_{n}\right\| \cdot\left\|x_{n+1}-q\right\| \\
=\left\|x_{n}-q\right\|^{2}-2 b_{n}^{N} \Phi\left(\left\|x_{n+1}-q\right\|\right)+o\left(b_{n}^{N}\right),
\end{aligned}
$$

where

$$
\begin{gathered}
2 b_{n}^{N}\left\|x_{n+1}-x_{n}\right\| \cdot\left\|x_{n+1}-q\right\|+2 b_{n}^{N}\left\|S_{N} x_{n+1}-S_{N} x_{n}^{N-1}\right\| \\
\cdot\left\|x_{n+1}-q\right\|+2 c_{n}^{N}\left\|u_{n}^{N}-x_{n}\right\| \cdot\left\|x_{n+1}-q\right\|=o\left(b_{n}^{N}\right) .
\end{gathered}
$$

By Lemma 2.3, we obtain $\lim _{n \rightarrow \infty}\left\|x_{n}-q\right\|=0$. This completes the proof.

Remark 3.2 Theorem 3.1 generalizes Theorem 3.1 of [1] and Theorem 2 of [2] in the following cases:

(a) It is not necessary for each range of $A_{i}$ or $I-A_{i}$ to be bounded in [1] and [2].

(b) The condition of $\left\{c_{n}^{i}\right\}$ is weakened to $c_{n}^{N}=o\left(b_{n}^{N}\right)$ from $\lim _{n \rightarrow \infty} \frac{c_{n}^{i}}{b_{n}^{i}+c_{n}^{i}}=0$ $(i=1,2, \ldots, N)$.

(c) The proof method of our theorem differs from that of [1] and [2].

Theorem 3.3 Let $E,\left\{u_{n}^{i}\right\},\left\{a_{n}^{i}\right\},\left\{b_{n}^{i}\right\},\left\{c_{n}^{i}\right\}(i=1,2, \ldots, N)$ be as in Theorem 3.1 and let $\left\{T_{i}\right\}_{i=1}^{N}: E \rightarrow E$ be $N$ uniformly continuous $\phi$-strongly pseudocontractive mappings. Then, for some $x_{0} \in E$, the multi-step iterative sequence with errors $\left\{x_{n}\right\}_{n=1}^{\infty}$ defined by

$$
\begin{aligned}
& x_{0} \in E, \\
& x_{n}^{1}=a_{n}^{1} x_{n}+b_{n}^{1} T_{1} x_{n}+c_{n}^{1} u_{n}^{1},
\end{aligned}
$$




$$
\begin{aligned}
& x_{n}^{2}=a_{n}^{2} x_{n}+b_{n}^{2} T_{2} x_{n}^{1}+c_{n}^{2} u_{n}^{2}, \\
& \vdots \\
& x_{n}^{N-1}=a_{n}^{N-1} x_{n}+b_{n}^{N-1} T_{N-1} x_{n}^{N-2}+c_{n}^{N-1} u_{n}^{N-1}, \\
& x_{n+1}=x_{n}^{N}=a_{n}^{N} x_{n}+b_{n}^{N} T_{N} x_{n}^{N-1}+c_{n}^{N} u_{n}^{N}, \quad n \geq 0,
\end{aligned}
$$

converges strongly to the unique common fixed point of $\left\{T_{i}\right\}_{i=1}^{N}$.

Proof See [1].

\section{Competing interests}

The authors declare that they have no competing interests.

\section{Authors' contributions}

All authors contributed equally in writing this paper, and read and approved the final manuscript.

\section{Author details}

'Department of Mathematics and Physics, Shijiazhuang Tiedao University, Shijiazhuang, 050043, China. ${ }^{2}$ Department of Mathematics, Shijiazhuang Mechanical Engineering College, Shijiazhuang, 050003, China.

\section{Acknowledgements}

The authors are very grateful to Professor Yeol-Je Cho for good suggestions which helped to improve the manuscript. This work is supported by the Hebei Natural Science Foundation No. A2011210033.

Received: 2 September 2012 Accepted: 6 January 2013 Published: 30 January 2013

\section{References}

1. Gurudwan, N, Sharma, BK: Convergence theorem for the common solution for a finite family of $\phi$-strongly accretive operator equations. Appl. Math. Comput. 217(15), 6748-6754 (2011)

2. Yang, L: A note on a paper 'Convergence theorem for the common solution for a finite family of $\phi$-strongly accretive operator equations.' Appl. Math. Comput. 218(21), 10367-10369 (2012)

3. Rafiq, A: On iterations for families of asymptotically pseudocontractive mappings. Appl. Math. Lett. 24(1), 33-38 (2011)

4. Deimling, K, Nonlinear Functional Analysis. Springer, Berlin (1985)

5. Moore, C, Nnoli, BVC: Iterative solution of nonlinear equations involving set-valued uniformly accretive operators. Comput. Math. Appl. 42(1-2), 131-140 (2001) 